\title{
Análise das condições de saúde e de vida da população urbana de Botucatu, São Paulo (Brasil). IV - Morbidade referida em entrevistas domiciliárias, 1983- 1984*.
}

\author{
Analysis of standards of living and health in the urban population of Botucatu, $S$. \\ Paulo State (Brazil). IV - Referred morbidity in household interviews, 1983-1984
}

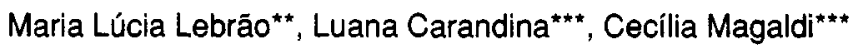

\begin{abstract}
LEBRÃO, M.L. et al. Análise das condições de saúde e de vida da população urbana de Botucatu, São Paulo (Brasil). IV-Morbidade referida em entrevistas domiciliárias, 1983-1984. Rev. Saúde públ., S. Paulo, 25: 452-60, 1991. Objetivou-se estudar a morbidade referida pela população urbana amostrada, no Município de Botucatu, SP, Brasil, em 1983/84, segundo sexo, idade, escolaridade e renda per capita. O método consistiu em entrevistas domiciliárias, com aplicação de dois formulários précodificados. Os entrevistadores eram leigos treinados e supervisionados, e a pessoa entrevistada foi quase sempre a mãe de familia. O período recordatório estabelecido em relação aos eventos informados (queixas, sintomas, acidentes comuns e diagnósticos) foi de três semanas. Das 7.075 pessoas amostradas ( $12 \%$ da população), $56 \%$ apresentaram episódios mórbidos, totalizando 6.649 episódios. As mulheres, bem como o grupo etário de 50 e mais anos apresentaram maior freqüência de queixas. A escolaridade e a renda per capita não diferenciaram o\$ entrevistados quanto à ocorrência maior ou menor de episódios. A prevalência de episódios mórbidos toi de 939/1.000 entrevistados. Predominaram queixas do aparelho respiratório (20\% do total de queixas), principalmente as infecçóes respiratórias agudas. Em segundo lugar, os sinais e sintomas mal definidos $(19 \%)$ e, a seguir, as doenças do sistema osteo-muscular, do sistema nervoso e do sistema circulatório, com proporções similares (ao redor de $9 \%$ ) e, finalmente, as do sistema digestivo $c$ as lesões e envenenamentos (ao redor de $8 \%$ ). Foram estimados os coeficientes de prevalência por grupos de doença (pela CID), segundo as variáveis estudadas. São comentadas as dificuldades de comparação dos resultados obtidos com os de trabalhos congêneres, face às diferenças nos métodos usados, apontando-se para a necessidade de uma padronização metodológica dos estudos de morbidade referida, cuja importância epidemiológica e para o planejamento em saúde vem sendo amplamente reconhecida.
\end{abstract}

Descritores: Morbidade. Entrevistas. Levantamentos epidemiológicos.

\section{Introdução}

A importância, para a epidemiologia e para o planejamento em saúde, dos inquéritos de morbidade referida, em populações, em que pesem suas dificuldades metodológicas e seu custo operacional, vem sendo cada vez mais reconhecida. Isto porque, ainda que necessárias, as informações obtidas dos serviços de saúde referem-se à

* Projeto financiado pela Financiadora de Estudos e Projetos (FINEP). Processo n 4.1.83.0232.00.

** Departamento de Epidemiologia da Faculdade de Saúde Pública da Universidade de São Paulo - São Paulo, SP - Brasil.

*** Departamento de Medicina Legal e Medicina em Saúde Pública da Faculdade de Medicina da Universidade Estadual Paulista - Botucatu, SP - Brasil.

Separatas/Reprints: M.L. Lebrão - Av. Dr. Arnaldo, 715 - 01255 - São Paulo, SP - Brasil.

Publicação financiada pela FAPESP - Processo Medicina $90 / 4602-1$ demanda e não à comunidade, podendo levar a distorções dos quadros de morbidade, em grupos populacionais. Além disso, os registros de dados de rotina nem sempre são completos e adequados, sob o ponto de vista quantitativo e qualitativo.

Avaliar a morbidade junto à população significa tentar descobrir o componente submerso do processo saúde/doença - a "morbidade percebida", ou "sentida" ou ainda "reprimida", sem acesso aos serviços, possibilitar a análise da determinação social desse processo e, por fim, subsidiar o planejamento de cuidados à saúde, em diferentes níveis geográficos ou administrativos. Outras vantagens adicionais desses inquéritos consistem na revelação, a entrevistadores leigos, das representações da população sobre saúde e morbi-mortalidade, das práticas usuais e não formais de prevenção e restauração da saúde, bem como do juizo crítico sobre os serviços ${ }^{3,15}$.

Instrumentos hoje bastante aplicados em investigações epidemiológicas sobre morbidade e utilização de serviços de saúde, a entrevista domiciliar 
e o questionário têm merecido avaliações de autores estrangeiros ${ }^{12,13,17,26}$, bem como de pesquisadores nacionais com experiência no campo ${ }^{7,10}$.

Segundo Kroeger ${ }^{19}$, esses instrumentos foram inicialmente empregados em centros industrializados e logo adaptados nos países em desenvolvimento, consoante as condições locais de sociedades com diferentes etnias.

A difusão progressiva da entrevista e do questionário, nestes últimos países, suscitou de Kroeger ${ }^{19}$, de Ross e Vaughan ${ }^{26}$, entre outros, exaustivas análises sobre os métodos usados e os resultados obtidos. Esses autores deram ênfase aos seguintes ítens: 1) desenho amostral da população a ser estudada; 2) controvérsia sobre a percepção de doença e validade de sua declaração pelas pessoas e, consequientemente, sobre a categorização de diagnósticos; 3) conteúdo do questionário; 4) período recordatório relativo aos eventos mórbidos referidos; 5) perfil e treinamento dos entrevistadores; 6 ) controle de erros nas respostas e 7) estimativas de índices de morbidade.

Ross e Vaughan ${ }^{26}$, revendo a literatura disponível, dedicaram sua avaliação aos trabalhos transversais realizados nos últimos 15 anos, em países menos desenvolvidos, e concluíram que os resultados são de difícil interpretação e de uso limitado, em vista da falta de padronização metodológica e/ ou de rigor em sua aplicação. Neste sentido, alertam para os cuidados na comparação de resultados obtidos em diferentes áreas e fazem recomendações que vão desde a definição clara de objetivos até à classificação das informações de morbidade e seu retorno à população de estudo.

Em vários países foram implantados sistemas de entrevistas para avaliar continuamente o estado de saúde e a utilização de serviços, destacando-se o dos Estados Unidos da América, o "National Health Interview Survey", cujos resultados vêm sendo publicados em séries do "Vital \& Health Statistics", desde $1963^{\prime}$. Outros trabalhos de vulto, nessa linha, são os de Kohn e White ${ }^{18}$, de White e col. ${ }^{28,29}$ em países da América e Europa e o de Laurell e col. ${ }^{20}$, no México.

$\mathrm{Na}$ literatura nacional, ainda escassa em estudos de morbidade referida, cumpre registrar o de Carvalheiro ${ }^{6}$, cujo sistema de colheita de dados, na população amostrada de Ribeirão Preto (SP), possibilitou a realização de diversas outras pesqui$\operatorname{sas}^{2,8,9,14,22,25.27}$

Em 1981, o Instituto Brasileiro de Geografia e Estatística (IBGE) realizou a Pesquisa Nacional por Amostragem de Domicílios, PNAD Saúde, em nove grandes capitais do país, apresentando taxas de

* Cesar, C.L.G. - Comunicação pessoal. morbidade total que foram questionadas por conta de possíveis problemas metodológicas ${ }^{11,23}$.

Carvalho e col. ${ }^{11}$ estudaram a morbidade total e a utilização de consultas médicas, em cinco populações do Estado da Bahia, em 1982-86, usando questões semelhantes às da PNAD-1981.

Outro trabalho com objetivos semelhantes é o de Medici ${ }^{23}$, que analisou dados da PNAD 1986, para as regiões brasileiras, com ênfase no Nordeste.

Há, ainda em desenvolvimento, o estudo de morbidade referida no ERSA de Itapecerica da Serra (SP)*.

A perspectiva de mudanças no Sistema de Saúde, a partir de 1983, com a progressiva integração e descentralização de atividades e serviços, no Município de Botucatu, já à época um centro médico de nítida abrangência regional, com a existência de uma Faculdade de Medicina e de vários outros serviços, ensejou a elaboração de um projeto visando à análise das condições de saúde e de vida da população urbana ${ }^{5}$.

Nessa óptica, impunha-se um estudo da morbidade e do consumo de serviços de saúde, cujo delineamento básico pudesse ser utilizado também em pesquisas específicas e cujos resultados servissem para subsidiar o planejamento do sistema local de saúde. Outra justificativa consistiu na eventual contribuição do conjunto de investigações de campo para ensino de epidemiologia e de saúde pública, no Curso de Graduação em Medicina e na Residência Médica.

O presente trabalho tem como objetivo estudar os eventos mórbidos referidos pela população urbana amostrada, segundo as variáveis sexo, idade, escolaridade e renda per capita, de maio de 1983 a abril de 1984, no Município de Botucatu, Estado de São Paulo.

\section{Material e Método}

A caracterização do munícipio, sua população e o desenho da amostra efetuada encontram-se referidos em publicação anterior ${ }^{5}$.

Foram utilizados como instrumentos dois formulários pré-codificados. O primeiro traz todas as informaçōes referentes ao núcleo familiar e a cada um de seus membros: idade, sexo, religião, grau de instrução, profissão, ocupação, renda individual e familiar e inserção no mercado de trabalho. $O$ segundo formulário específico para cada problema, diz respeito aos episódios de doença e/ou acidente comum, sua duração e as possiveis sequielas dos acidentes, ocorridos ou existentes no período recordatório, a conduta adotada em cada episódio e a utilização de serviços. Esses ítens foram abordados por meio de perguntas abertas. Os acidentes do 
trabalho foram objeto de estudo específico, com formulário próprio e período recordatório de 12 meses, sendo a pessoa entrevistada o próprio acidentado, não estando portanto incluídos no presente trabalho.

A metodologia consistiu na aplicação dos dois formulários previamente elaborados e testados, visando à obtenção da morbidade referida por pessoas de ambos os sexos e de todas as idades, ao longo de 12 meses consecutivos de levantamento, no período de maio de 1983 a abril de 1984 , abrangendo desta forma os episódios mórbidos que apresentam variações ou exacerbações sazonais.

Foram definidos como episódios mórbidos todas as queixas, sintomas, acidentes comuns e diagnósticos referidos. As entrevistas foram realizadas nos últimos sete dias de cada mês, tendo sido definido como período de estudo o intervalo entre o início do mês e o dia de entrevista. Dessa forma, o período recordatório ultrapassou os quinze dias preconizados pelos principais autores ${ }^{19,26}$ variando de 24 a 30 dias (em média três semanas). Tal fato se deveu a ter-se verificado que o período recordatório é menos impreciso quando referido ao começo do mês e não a qualquer data. A não adoção da terceira semana para a realização das entrevistas decorreu da grande concentração de feriados nesse período, no ano de estudo.

Foi estabelecido que o informante seria a mãe da família e, apenas na sua ausência após três tentativas, o chefe da família e, em última instância, outro elemento adulto responsável e conhecedor das informações familiares previstas nos dois formulários. Foi considerado financeira e operacionalmente inviável conduzir-se as entrevistas com cada um dos elementos envolvidos.

O uso de entrevistadores foi necessário em razão do nível de instrução da maior parte da população amostrada. Optou-se pelo menor número possível de entrevistadores a fim de reduzir a quantidade de erros que pudessem ser introduzidos, mesmo que esse fato tenha levado a uma ampliação do período de realização das entrevistas.

Os entrevistadores foram selecionados entre pessoas da própria comunidade, com bom grau de instrução e leigos em saúde. Os mesmos receberam treinamento teórico-prático e instruções por escrito visando ao correto preenchimento dos formulários e à adequada abordagem do entrevistador. Sua atuação foi permanentemente avaliada por dois supervisores de campo que também faziam a revisão de todos os formulários preenchidos.

No presente trabalho, os episódios mórbidos referidos foram analisados segundo as variáveis: sexo, idade, escolaridade e renda per capita. $\mathrm{O}$ item ocupação não foi estudado tendo em vista que $67 \%$ dos entrevistados não estavam inseridos na força de trabalho (menores de sete anos, estudantes, aposentados, pensionistas e donas de casa) e os restantes $33 \%$ mostraram grande dispersão entre as categorias ocupacionais.

Os dados obtidos foram codificados segundo manual previamente elaborado e os episódios mórbidos pelas subcategorias da Classificação Internacional de Doenças, Revisão de $1975^{21}$, por pessoal especialmente treinado. A seguir, esses dados foram processados eletronicamente pelo programa SPSS (Statistical Package of Social Science).

Tendo em vista que os dados nas tabelas se referem sempre à mesma fonte e ao mesmo período, essa informação será omitida no título das mesmas.

A análise dos dados limitou-se à elaboração de proporções e coeficientes.

\section{Resultados e Discussão}

Foram entrevistadas 1.769 famílias, correspondendo a um total de 7.075 pessoas, das quais 3.967 apresentaram pelo menos um episódio mórbido $(56,1 \%)$, tendo sido referidos, no total, 6.649 episódios.

Em relação ao sexo, os dois grupos (com ou sem episódio mórbido) tiveram as distribuições que podem ser vistas na Tabela 1.

Tabela 1. Distribuição (№ e \%) dos entrevistados com ou sem episódios mórbidos, segundo o sexo.

\begin{tabular}{ccccccc}
\hline Sexo & \multicolumn{5}{c}{ Episódio } & \multicolumn{2}{c}{ Total } \\
& $\mathrm{N}^{2}$ & $\%$ & $\mathrm{~N}^{2}$ & $\%$ & $\mathrm{~N}^{2}$ & $\%$ \\
\hline Masculino & 1.786 & 45,0 & 1.689 & 54,4 & 3.475 & 49,1 \\
& $(51,4 \%)$ & & $(48,6 \%)$ & & $(100,0 \%)$ & \\
Feminino & 2.181 & 55,0 & 1.415 & 45,6 & 3.596 & 50,9 \\
& $(60,7 \%)$ & & $(39,3 \%)$ & & $(100,0 \%)$ & \\
\hline Total & $3.967^{\star}$ & 100,0 & 3.104 & 100,0 & $7.071^{*}$ & 100,0 \\
& $(56,1 \%)$ & & $(43,9 \%)$ & & $(100,0 \%)$ & \\
\hline
\end{tabular}

* Faltam 4 pessoas, sem informaçáo do sexo.

Um aspecto que costuma ser ressaltado nas publicações a respeito dos inquéritos domiciliares de morbidade e que pode ser atribuído à metodologia e ao comportamento dos entrevistados é a referência de queixas segundo sexo. A Tabela 1 mostra que as mulheres apresentaram queixas mais freqüentemente do que os homens. Isto pode ser visto tanto quando se considera a participação das mulheres entre as pessoas com episódios mórbidos como quando se considera as mulheres com episódios entre o total de mulheres, num percentual muito superior ao verificado entre os homens. Dois pontos podem ser levantados em relação a esta questão: o primeiro diz respeito ao efeito da resposta delegada, na medida em que ela pode provocar uma subenumeração das queixas de outras pessoas, o que explicaria o maior 
número de mulheres com queixas ${ }^{26}$. Por outro lado, várias publicações referem que as mulheres apresentam queixas com maior freqüência ${ }^{2,6,11,16,23}$.

A Tabela 2 mostra a distribuição dos dois grupos segundo as faixas etárias, onde se observa, conforme esperado, que os grupos de menor e maior proporção de positividade em relação às queixas foram respectivamente os de 5 a 20 anos e o de 50 anos e mais. O primeiro por ser um grupo sabidamente de baixa morbidade e mortalidade ${ }^{16}$ e o segundo pelos problemas inerentes à idade. Dados da literatura corroboram esses achados ${ }^{2,6,11,23}$.

Tabela 2. Distribuição dos entrevistados ( $N^{Q}$ e \%) com ou sem episódios mórbidos, segundo a faixa etária.

\begin{tabular}{lrrrrrr}
\hline \multirow{2}{*}{ Faixa etária } & \multicolumn{3}{c}{ Episódio } & \multicolumn{2}{c}{ Com } & \multicolumn{2}{c}{ Sem } & \multicolumn{2}{c}{ Total } \\
& $N^{2}$ & $\%$ & \multicolumn{1}{c}{$N^{2}$} & $\%$ & \multicolumn{1}{c}{$\mathrm{N}^{2}$} & $\%$ \\
\hline$<1$ ano & 84 & 60,4 & 55 & 39,6 & 139 & 100,0 \\
$1 \vdash 5$ anos & 394 & 57,8 & 288 & 42,2 & 682 & 100,0 \\
$5 \vdash 20$ anos & 979 & 47,1 & 1.099 & 52,9 & 2.078 & 100,0 \\
$20 \vdash 50$ anos & 1.492 & 51,1 & 1.428 & 48,9 & 2.920 & 100,0 \\
50 anos e + & 1.020 & 81,3 & 234 & 18,7 & 1.254 & 100,0 \\
\hline Total & 3.969 & 56,1 & 3.104 & 43,9 & 7.073 & 100,0 \\
\hline
\end{tabular}

A Tabela 3 mostra a distribuição dos dois grupos segundo escolaridade.

Tabela 3. Distribuição dos entrevistados (№ e \%) com ou sem episódios mórbidos, segundo o grau de escolaridade.

\begin{tabular}{lrrrrrrr}
\hline \multirow{2}{*}{ Escolaridade } & \multicolumn{3}{c}{ Episódio } & \multicolumn{3}{c}{ Com } & \multicolumn{2}{c}{ Sem } & \multicolumn{2}{c}{ Total } \\
& $N^{0}$ & $\%$ & $N^{2}$ & $\%$ & $N^{2}$ & $\%$ \\
\hline$<7$ anos & 654 & 56,5 & 504 & 43,5 & 1.158 & 100,0 \\
analfabeto & 308 & 81,3 & 71 & 18,7 & 379 & 100,0 \\
até 4 ${ }^{2}$ s.incompl. & 936 & 63,6 & 536 & 36,4 & 1.472 & 100,0 \\
até col.incompl. & 1.575 & 52,2 & 144 & 47,8 & 3.020 & 100,0 \\
até sup.incompl. & 311 & 44,7 & 385 & 55,3 & 696 & 100,0 \\
superior completo & 157 & 50,5 & 154 & 49,5 & 311 & 100,0 \\
s/informação & 29 & 76,3 & 9 & 23,7 & 38 & 100,0 \\
\hline Total & 3.970 & 56,1 & 3.104 & 43,9 & 7.074 & 100,0 \\
\hline
\end{tabular}

Chama a atenção, entre os agrupamentos, a elevada proporção dos analfabetos com queixas em relação ao total dos analfabetos. A distribuição desta categoria segundo os grupos etários mostrou que $69,0 \%$ tinha 50 anos e mais, o que poderia explicar a maior frequiência de queixas encontradas. Registre-se que dos analfabetos com 50 anos e mais $72,9 \%$ são mulheres.

A variável renda per capita não diferenciou os entrevistados quanto à presença maior ou menor de episódios mórbidos (Tabela 4). Carvalheiro ${ }^{6} \mathrm{em}$ Vila Lobato, encontrou resultado semelhante. Kroeger $^{19}$ salienta as controvérsias relacionadas à informação relativa à renda, nos países em desenvolvimento, o que traz problemas para relacioná-la adequadamente com o estado de saúde.
Tabela 4. Distribuição dos entrevistados (№ e \%) com ou sem episódios mórbidos segundo a renda per capita (em salários mínimos).

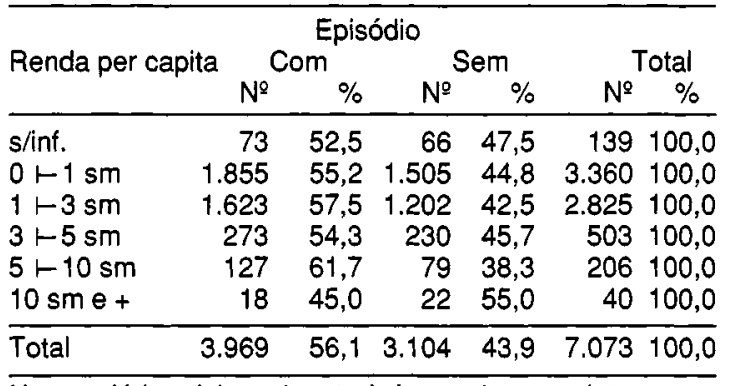

Nota: salário mínimo vigente à época da entrevista.

O total de episódios mórbidos referidos pelos entrevistados foi de 6.648 queixas, resultando num coeficiente de prevalência de 939,6 episódios por mil entrevistados no período (Tabela 5). Os coeficientes encontrados em outros estudos $2,6,18,20,26,29$ mostraram valores variando de 200 episódios/mil pessoas/mês a cerca de $700 / \mathrm{mil}$ pessoas/mês. Segundo Ross e Vaughan ${ }^{26}$ e Kroeger ${ }^{19}$ essa grande diversidade deve-se, fundamentalmente, a problemas metodológicos, além das reais diferenças que possam existir nos grupos estudados. A falta de padronização prejudica a comparação entre o conjunto dos trabalhos.

A Tabela 5 apresenta detalhamento dessas queixas, segundo o seu agrupamento em capítulos da CID. Em primeiro lugar surgem as doenças do aparelho respiratório, com o coeficiente de 195,3/mil entrevistados, representando $20,8 \%$ do total das queixas. Esse resultado não surpreende, na medida em que as doenças do aparelho respiratório geralmente predominam em estudos de morbidade, tanto por inquéritos domiciliares ${ }^{6,8,26}$ como nos estudos das demandas hospitalares e ambulatoriais ${ }^{4,8,24}$. Dentre as doenças do aparelho respiratório, destacam-se com grande peso as doenças respiratórias agudas, como as gripes e resfriados, que sozinhas representaram $14,2 \%$ do total das queixas, ou seja, $67,8 \%$ das respiratórias. Os maiores coeficientes dessas queixas são encontrados nos menores de 5 anos, diminuindo nos grupos etários subseqüentes (Tabela 6). Os coeficientes por sexo apontam diferença apenas entre os menores de um ano, sem que se conheça fundamento epidemiológico para o fato. Por outro lado, as respiratórias crônicas apresentam seu menor coeficiente no grupo etário de 20 a 50 anos, o que poderia ser explicado pelo fato de estarem aí incluídas a asma (de aparecimento em idades mais jovens) e as bronquites crônicas, que afetam idades mais avançadas (Tabela 6). Quando se somam as tosses (sinais e sintomas mal definidos) às doenças respiratórias crônicas, acentua-se a preponderância da faixa de 50 anos e mais, elevando- 
Tabela 5. Distribuiçā.s dos episódios mórbidos ( $N^{2}, \%$ e coef.) segundo os capítulos (e destaques) da CID.

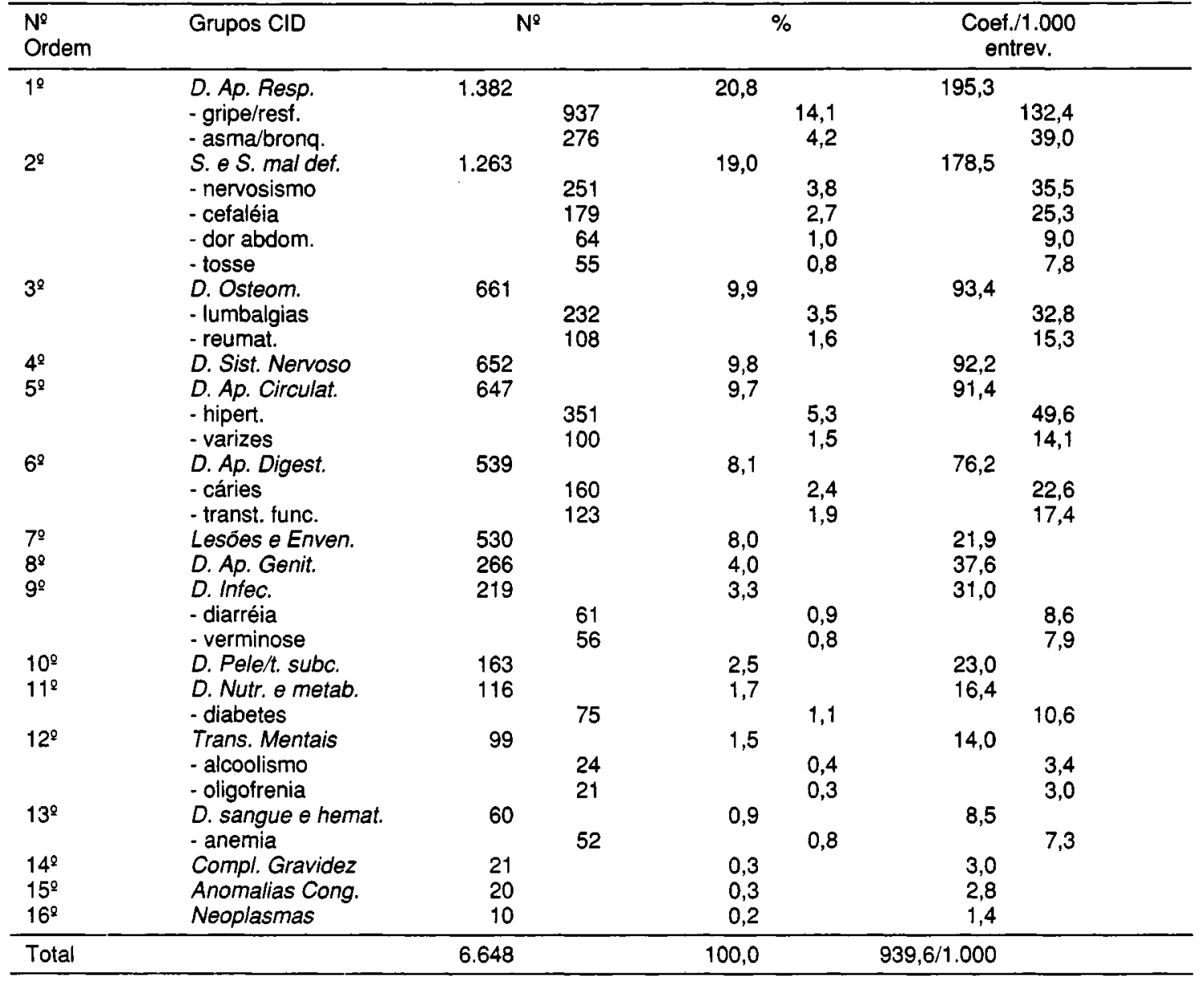

se de 56,9/mil entrevistados para 77,0/mil entrevistados, embora o caráter crônico dessas tosses não esteja claro nas respostas dos entrevistados.

Nos países em desenvolvimento, os sinais e sintomas mal definidos costumam ocupar lugar de destaque, especialmente quando as informações são obtidas através de inquéritos domiciliares em que os informantes são leigos ${ }^{8,26}$. No presente estudo eles ocupam a segunda posição, com coeficiente de $178,5 /$ mil pessoas correspondendo a $19,0 \%$ do total das queixas.

Nervosismo, cefaléia, dor abdominal e tosse, nessa ordem, foram os mais frequientes. Por grupo etário, esse capítulo apresenta seu maior coeficiente entre os de 50 anos e mais principalmente no sexo feminino. Sabidamente os idosos, em especial as mulheres, referem mais queixas que dificilmente são traduzidas em diagnósticos sindrômicos. Em relação ao nervosismo verifica-se que as mulheres apresentam esse sintoma em número muito maior do que os homens $(50,9 / 1.000$ entrevistadas/19,6/ 1.000 entrevistados), com predominância da faixa etária de 20 a 50 anos. Da mesma maneira se com- portam a cefaléia e a dor abdominal, isto é, foram mais freqüentes em mulheres de 20 a 50 anos.

Os capítulos das doenças respiratórias e dos sinais e sintomas mal definidos juntos representaram quase $40 \%$ das queixas, tendo exibido comportamento semelhante no estudo de Ribeirão Preto ${ }^{6}$.

Os capítulos seguintes - doenças osteo-musculares, doenças do sistema nervoso, doenças de aparelho circulatório aparecem com proporções bastante semelhantes (ao redor de $9,8 \%$ ), podendo ser considerados como pertencentes ao terceiro posto. Dentre as doenças osteo-musculares, ressaltam as lumbalgias, com 3,5\% do total das queixas, principamente a partir da idade adulta e no sexo feminino. Esse resultado pode ser explicado pelas condições desfavoráveis em que atua a força de trabalho e pelo fato de as informações terem sido prestadas pelas donas de casa cujas tarefas domésticas favorecem esse tipo de sintoma.

Segundo a Tabela 6 , as queixas do sistema nervoso concentram-se nas idades acima de 20 anos e no sexo feminino, relacionando-se mais com os distúrbios da visão. 


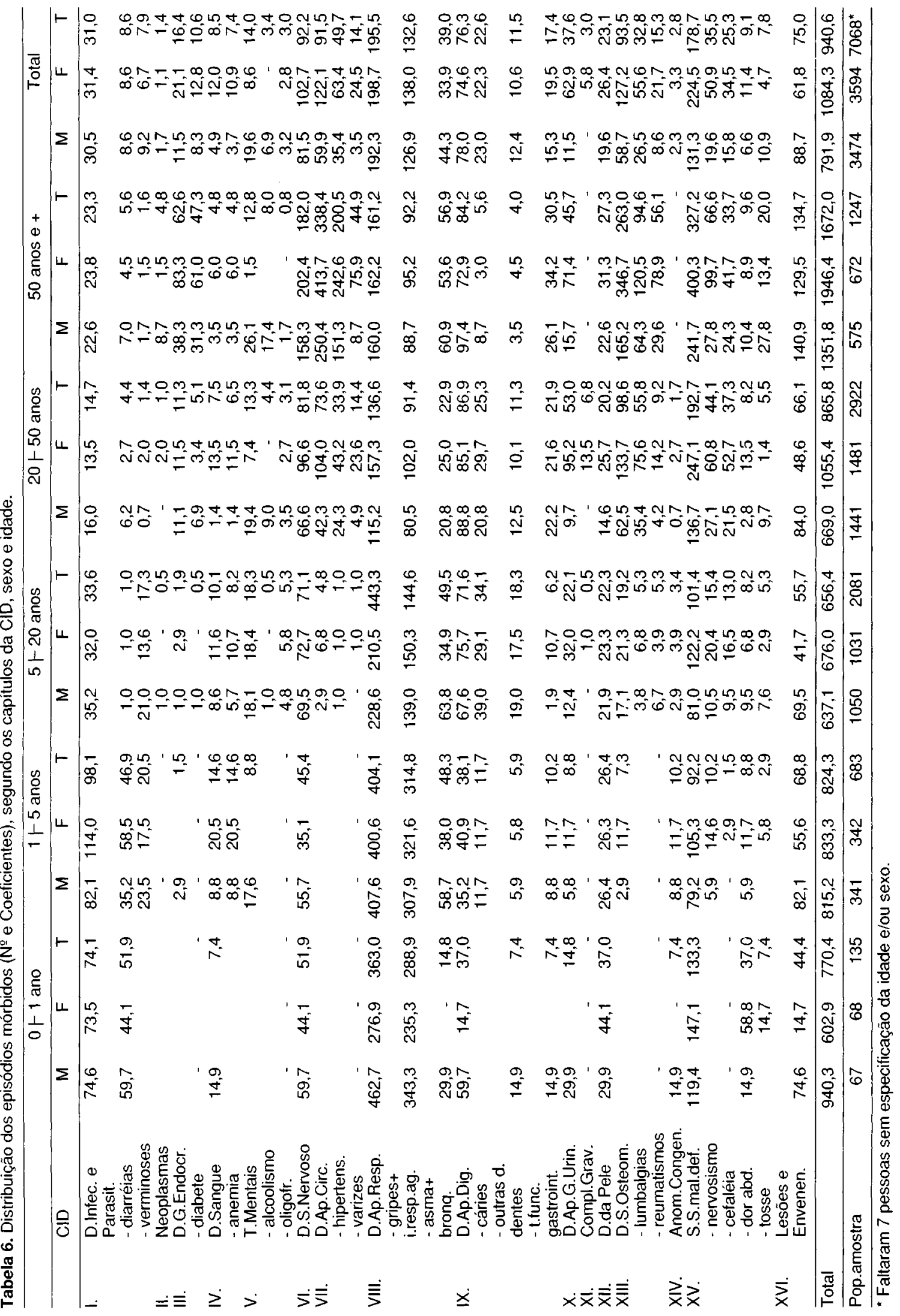


Em relação às doenças do aparelho circulatório, a hipertensão arterial representou 5,3\% do total de queixas referidas (Tabela 5) sendo cinco vezes maior o coeficiente quando comparado o grupo de 50 anos e mais com o de 20 a 50 anos, e o do sexo feminino, sempre maior do que o masculino (Tabela 6). Estudo sobre hiperteryão realizado posteriormente com a mesma população e complementado com as medidas da pressão arterial mostrou coeficientes de prevalência de 123/mil pessoas acima de 16 anos, contra 49,6/mil entrevistados deste estudo*.

O capítulo de doenças que segue é o do aparetho digestivo, cujo coeficiente foi de $76,2 / \mathrm{mil}$ pessoas aumentando com a idade e representando $8,1 \%$ do total de queixas, destacando-se as referências às cáries e aos transtornos funcionais (Tabelas 5 e 6).

Em outros trabalhos ${ }^{6,8,26}$, as doenças do aparelho digestivo têm ocupado as primeiras posições, porém sem a especificação dos principais problemas deste capítulo.

Finalmente, entre os capítulos que merecem referência, cita-se "Lesões e Envenenamentos" com o coeficiente de $21,9 /$ mil pessoas e representando $8 \%$ do total de queixas. Nesse capítulo os homens apresentaram maior número de episódios, sugerindo que dada a evidência desse tipo específico de agravo, a resposta delegada (proxy-responding) parece interferir menos que nos demais.

Carvalheiro $0^{6,8}$ encontrou resultados percentuais semelhantes para esse capítulo em Vila Lobato e Vila Guatapará, enquanto em Ribeirão Preto esse valor foi de apenas $2,0 \%$.

Os demais capítulos têm freqüências baixas, totalizando apenas $14,7 \%$ das queixas. Vale ressaltar a pouca representatividade das doenças infecciosas $(3,3 \%)$ quando comparadas aos outros trabalhos em países em desenvolvimento.

Em relação à escolaridade, os episódios mórbidos segundo os capítulos da CID apresentaram os coeficientes constantes da Tabela 7. Repetindo o que foi visto anteriormente, em relação à Tabela 3 , os analfabetos apresentaram o maior coeficiente de queixas (1.775,7/mil analfabetos). Essa mesma situação se repete nos capítulos relativos a sinais e sintomas mal definidos, doenças do sistema osteomuscular, doenças do aparelho circulatório e lesões e envenenamentos. Em grau menor, o mesmo acontece em relação aos mesmos capítulos com o grupo de escolaridade até quarta série incompleta. A supasição possível de que o baixo nível de escolaridade esteja associado com piores condições de vida, que se expressariam através de um maior coe-

Tabela 7. Distribuição dos coeficientes (por mil pessoas) dos episódios mórbidos, segundo os capítulos da CID e a escolaridade dos entrevistados.

\begin{tabular}{|c|c|c|c|c|c|c|c|}
\hline Capítulos da CID & $<7$ a. & \multicolumn{3}{|c|}{ Grau de Escolaridade } & $\begin{array}{l}\text { até super. } \\
\text { inc. }\end{array}$ & $\begin{array}{l}\text { super. } \\
\text { comp. }\end{array}$ & Total* \\
\hline $\begin{array}{l}\text { Doenças Apar.Resp. } \\
\text { Sinais Sintomas }\end{array}$ & 359,2 & 163,6 & 220,1 & 142,4 & 139,4 & 157,6 & 195,8 \\
\hline $\begin{array}{l}\text { mal def. } \\
\text { Doenças Osteo-musc. e }\end{array}$ & 93,3 & 393,1 & 222,8 & 181,1 & 122,1 & 131,8 & 178,8 \\
\hline $\begin{array}{l}\text { Tec. Conj. } \\
\text { Doenças Sist.Nerv. e }\end{array}$ & 7,8 & 300,8 & 120,9 & 94,0 & 73,3 & 77,2 & 93,8 \\
\hline órgāos sent. & 49,2 & 163,6 & 116,8 & 91,7 & 63,2 & 109,3 & 91,8 \\
\hline Doenças Ap. Circ. & & $\begin{array}{r}345,6 \\
95,0\end{array}$ & $\begin{array}{r}146,1 \\
78.1\end{array}$ & $\begin{array}{l}84,8 \\
80.5\end{array}$ & $\begin{array}{l}37,4 \\
89.1\end{array}$ & $\begin{array}{l}45,0 \\
93,2\end{array}$ & $\begin{array}{l}91,2 \\
76.5\end{array}$ \\
\hline $\begin{array}{l}\text { Doenças Ap.Digest. } \\
\text { Lesóes e Env. } \\
\text { Doenças Ap.Genito- }\end{array}$ & $\begin{array}{l}45,8 \\
69,1\end{array}$ & $\begin{array}{r}95,0 \\
137,2\end{array}$ & $\begin{array}{l}78,1 \\
84,2\end{array}$ & $\begin{array}{l}80,5 \\
76,2\end{array}$ & $\begin{array}{l}89,1 \\
35,9\end{array}$ & $\begin{array}{l}93,2 \\
57,9\end{array}$ & $\begin{array}{l}76,5 \\
75,2\end{array}$ \\
\hline $\begin{array}{l}\text { Urin. } \\
\text { Doenças Infec. e }\end{array}$ & 11,2 & 47,5 & 34,6 & 49,7 & 28,7 & 38,6 & 37,5 \\
\hline $\begin{array}{l}\text { Parasit. } \\
\text { Doenças Pele e tec. }\end{array}$ & 88,1 & 15,8 & 31,9 & 15,6 & 12,9 & 19,3 & 30,8 \\
\hline Cel.Sub. & 26,8 & 21,1 & 23,8 & 21,2 & 24,4 & 25,7 & 23,2 \\
\hline Doenças G. Endocr. & 2,6 & 52,8 & 25,8 & 12,2 & 14,4 & 19,3 & 16,2 \\
\hline Transt.Mentais & 10,4 & 21,1 & 18,3 & 11,2 & 4,3 & 9,6 & 12,4 \\
\hline Doenças Sangue & 12,1 & 7,9 & 10,9 & 7,3 & 2,9 & 6,4 & 8,4 \\
\hline Compl.Gravidez & - & 2,6 & 2,0 & 3,6 & 5,7 & 6,4 & 3,0 \\
\hline Anom.Congen. & 9,5 & 2,6 & 2,0 & 0,7 & 1,4 & 6,4 & 2,8 \\
\hline Neoplasmas & - & 5,3 & 2,0 & 1,3 & 1,4 & - & 1,4 \\
\hline Total & 785,0 & $1.775,7$ & $1.140,6$ & 873,5 & 656,6 & 803,8 & 938,9 \\
\hline Pop.amostra & 1.158 & 379 & 1.472 & 3.020 & 696 & 311 & $7.036^{* *}$ \\
\hline
\end{tabular}

* Foram excluídas do total, em cada capítulo, as queixas sem informação da escolaridade (total de 42 queixas).

* Foram excluídos 38 casos, sem informação da escolaridade, do total de 7.074 pessoas.

* Habermann, F. - Relatório Projeto FINEP - Dados Inéditos. 
ficiente de morbidade, maior exposição aos efeitos de ocupações pouco qualificadas porém de maior esforço físico, de maior exposição aos acidentes e maior grau de estresse, fica prejudicada, conforme comentário anterior, pela predominância de pessoas maiores de 50 anos e de mulheres entre os entrevistados com ausência ou baixa escolaridade.

Além disso, a impossibilidade de associação neste trabalho, da escolaridade com a ocupação e a restrição da análise a cada variável sócio-econômica, não permitem avaliar a hipótese anteriormente levantada. Recentemente, autores, como Barros ${ }^{2}$, vêm tentando utilizar classe social ao invés de estratos sócio-econômicos constituídos pelas variáveis citadas para melhor avaliar a influência da qualidade de vida na saúde da população.

Conforme já comentado em relação à Tabela 4, a variável renda per capita analisada isoladamente, no presente estudo, não se mostrou discriminatória, também em relação aos capítulos da CID, como mostra a Tabela 8 .

\section{Considerações Gerais}

O presente trabalho evidencia algumas limitações metodológicas que dificultam a análise comparativa com trabalhos semelhantes de outras localidades. Poder-se-ia citar como exemplo a questão do informante que freqüentemente é uma única pessoa responsável pelo conjunto familiar, o que traz a possibilidade de subenumeração dos episódios mórbidos. Uma questão maior se refere à conceituação das questões formuladas, o que pode levar à impossibilidade de comparações consistentes, como por exemplo o que vem a ser "episódio mórbido". Esta questão se complica nu momento da classificação desses episódios. As variáveis sócio-econômicas também padecem da incomparabilidade, na medida em que cada trabalho realizado apresenta variáveis diferentes abordadas de diferentes maneiras, além de não permitirem uma visão global da qualidade de vida, na medida em que acabam sendo analisadas individualmente.

Por tudo isso é evidente a necessidade de uma padronização metodológica desse tipo de levantamento epidemiológico que, como foi salientado desde a introdução, possibilita o diagnóstico das condições de saúde da população geral e não apenas de demandas de serviços, contribuindo para a análise da determinação social do processo saúde-doença e podendo subsidiar o planejamento em saúde.

\section{Agradecimentos}

Ao Sr. Fernão Dias de Lima, pelo seu empenho na recuperação do banco de dados, sem o que seria impossível realizar esse trabalho; às Sras. Rosângela
Tabela 8. Distribuição dos coeficientes (por mil pessoas) dos episódios mórbidos, segundo capítulos da CID e a renda per capita dos entrevistados.

\begin{tabular}{|c|c|c|c|c|c|}
\hline \multirow[b]{2}{*}{ Capítulos CID } & \multicolumn{5}{|c|}{ Renda per capita } \\
\hline & $\begin{array}{c}\text { sem } \\
\text { renda } \\
\text { e até } \\
1 \text { s.m. }\end{array}$ & $\begin{array}{l}\text { 1 a } 3 \\
\text { s.m. }\end{array}$ & $\begin{array}{l}3 \text { a } 5 \\
\text { s.m. }\end{array}$ & $\begin{array}{l}5 \mathrm{e}+ \\
\text { s.m. }\end{array}$ & $\begin{array}{c}\text { Total } \\
(*)\end{array}$ \\
\hline Doenças Ap.Resp. & 200,6 & 189,4 & 176,9 & 207,3 & 194,4 \\
\hline $\begin{array}{l}\text { Sint., Sinais } \\
\text { e afec.mal defin. } \\
\text { Doencas Osteo-Musc. }\end{array}$ & 181,0 & 187,6 & 161,0 & 126,0 & 180,0 \\
\hline $\begin{array}{l}\text { e do Tec.Conjunt. } \\
\text { Doenças Sist.Nervoso }\end{array}$ & 91,7 & 97,7 & 75,5 & 130,1 & 94,3 \\
\hline e O. dos Sentidos & 83,9 & 98,4 & 109,3 & 97,6 & 92,2 \\
\hline Doenças Ap.Circulat. & 88,1 & 99,8 & 77,5 & 73,2 & 91,6 \\
\hline Doenças Ap.Digestivo & 75,6 & 78,2 & 83,5 & 69,1 & 77,0 \\
\hline Lesões e Enven. & 76,5 & 76,1 & 67,6 & 73,2 & 75,6 \\
\hline Doenças Ap.Gen.Urin. & 30,6 & 42,8 & 41,7 & 65,0 & 37,6 \\
\hline Doenças Infec. Parasit. & 34,5 & 29,0 & 19,9 & 24,4 & 30,9 \\
\hline Cel.Subc. & 24,4 & 23,0 & 25,8 & 8.1 & 23,4 \\
\hline Doenças G.Endoc. & 12,8 & 17,7 & 27,8 & 24,4 & 16,3 \\
\hline Transt.Mentais & 14,9 & 12,7 & 15,9 & 16,3 & 14,1 \\
\hline Doenças Sangue e & & & & & \\
\hline Órgãos Hemat. & 11,3 & 5,7 & 8,0 & 4,1 & 8,5 \\
\hline Comp.Gravidez & 2,1 & 5,0 & - & & 3,0 \\
\hline Anom.Congenitas & 1,8 & 3,5 & 2,0 & 12,2 & 2,9 \\
\hline Neoplasmas & 0,9 & 2,1 & 2,0 & & 1,4 \\
\hline Total & 930,6 & 968,8 & 894,6 & 930,9 & 958,9 \\
\hline Pop. amostra & 3.360 & 2.825 & 503 & 246 & $6.934^{\star}$ \\
\hline
\end{tabular}

* Foram excluídas 139 pessoas sem informação de renda per capita.

* Foram excluídas do total, em cada capítulo, as queixas sem informação da renda per capita (total: 109 queixas).

Maria Giarola, Maria de Lourdes Silveira, Natalina de Castro Meneghini, Maria Luiza Neris e Mariângela Baldini Levy, que tornaram possível a execução de campo, classificação e processamento deste trabalho.

LEBRÃO, M.L. et al. [Analysis of standards of living and health in the urban population of Botucatu, S. Paulo State (Brazil). IV - Referred morbidity in household interviews, 1983-1984]. Rev. Saúde públ., S. Paulo, 25: $452-60,1991$. The objective of the study was to analyse the referred morbidity of the urban population resident in the city of Botucatu, S. Paulo State, Brazil for the period 1983-1984 was analysed. Data was obtained from a sample population of 7,075 persons ( 12 per cent of the population) by means of household interviews using two pre-codified questionnaires applied by trained lay interviews under supervision. The recall period of the events was fixed at three weeks. The variables studied were: sex, age, schooling, "per capita" income, and referred morbidity (complaints, symptoms, common accidents, and diagnoses). The results showed that 56 percent reported episodes of illness to a total of 6,649 . Women of 50 or more years old presented the highest frequency of complaints. There was no variation of occurrence of episodes in terms of "per capita" income. The prevalence of 
illness episodes was 939 per thousand persons. There was a predominance of complaints relating to the respiratory system ( 20 per cent of complaints), mainly acute respiratory infections; on a second level was signs, symptoms and ill-defined conditions (19 per cent), followed by diseases of the musculoskeletal system, of the nervous system and of the circulatory system (about $9 \%$ ) and, finally, diseases of the digestive system and lesions and poisoning ( $8 \%$ ). Specific rates of prevalence according to groups of diseases (ICD) and to the variables of study were estimated. The difficulties of comparing these results with those given by other works, because of the lack of homogeneity in the criteria adopted for obtaining information, are commented on. It is concluded that there is a need for a standardized procedure in any study of referred morbidity because of its epidemiological importance and its use in health planning.

Keywords: Morbidity. Interviews. Health surveys.

\section{Referências Bibliográficas}

1. ADAMS, P.F. \& BENSON, V. Current estimates from the national health interview survey, 1989. Vital Hlth Statist Ser. 10 (176) 1990.

2. BARROS, M.B.A. Saúde e classe social: um estudo sobre morbidade e consumo de medicamentos. Ribeirão Preto, 1983. [Tese de Doutoramento - Faculdade de Medicina de Ribeirão Preto da USP].

3. BARROS, M.B.A. \& CARVALHEIRO, J. da R. Entrevistas domiciliárias e o ensino e pesquisa em epidemiologia. Rev. Saúde públ., S.Paulo, 18: 411-7. 1984.

4. CALIXTO, A.G.Z. Atenção primária à saúde: a avaliação da resolutividade médica de um centro municipal de saúde de Botucatu - SP. Ribeirão Preto, 1990. [Dissertação de Mestrado - Faculdade de Medicina de Ribeirão Preto da USP].

5. CARANDINA, L.; SANCHES, O.; CARVALHEIRO, J, da $R$. Análise das condições de saúde e de vida da população urbana de Botucatu, SP. I - Descrição do plano amostral e avaliação da amostra. Rev. Saúde públ., S. Paulo, 20: 46574, 1986.

6. CARVALHEIRO, J. da R. Levantamento de condições de saúde por entrevistas domiciliárias. Ribeirão Preto, 1975. [Tese de Livre-Docência - Faculdade de Medicina de Ribeirão Preto da USP].

7. CARVALHEIRO, J. da R. \& SANCHES, O. Amostragem domiciliar contínua em estudos epidemiológicos e no ensino. Rev. Saúde públ., S. Paulo, 13: 195-202, 1979.

8. CARVALHEIRO, J. da R. \& CARVALHEIRO, C.D.G. Medidas de morbidade produzidas por duas fontes diversas, Ribeirão Preto, SP (Brasil), 1975. Rev. Saúde públ., S. Paulo, 13: 265-70, 1979.

9. CARVALHEIRO, J. da R.; CARVALHEIRO, C.D.G.; XAVIER, A.R.; COSTA, J.C. da. Contribuição das doenças infecciosas e parasitárias na morbi-mortalidade de Ribeirão Preto, S.P. (Brasil). Rev. Saúde públ., S. Paulo, 13: 203-7, 1979

10. CARVALHEIRO, J. da R. Investigação epidemiológica e entrevistas domiciliárias. Rev. Saúde públ., S. Paulo, 15: 54350, 1981 .

11. CARVAlHO, F.M.; SILVANY NETO, A.M.; PAIM, J.S.; MELO, A.M.C.; AZARO, M.G.A. Morbidade referida e utilização de consulta médica em cinco populações do Estado da Bahia. Ciênc. e Cult., 40: 853-8, 1988.

12. COBB, S. \& ROSENBAUM, J. A comparison of specific symptom data obtained by nonmedical interviewers and by physicians. J. chron. Dis., 4: 245-52, 1956.

13. FELDMAN, J.J. The household interview survey as a technique for the collection of morbidity data. J. chron. Dis., 11: 535$57,1960$.

14. FORSTER, A.C. Cobertura dos serviços de imunização de Ribeirão Preto através de entrevistas domiciliares. Ribeirão Preto, 1979. [Dissertação de Mestrado - Faculdade de Medicina de Ribeirão Preto da USP].

15. FUNDAÇÃO DO DESENVOL VIMENTO ADMINISTRATIVO (FUNDAP). Seminário interinstitucional sobre pesquisas de morbidade. Texto-base. São Paulo, 1984. [Mimeografado].

16. GUEDES, J. da S. Contribuição para o estudo da evolução do nível de saúde do Estado de São Paulo: análise das regiões administrativas (1950-1970). São Paulo, 1972. [Tese de Doutoramento - Faculdade de Saúde Pública da USP]

17. HANSON, R.H. \& MARKS, E.S. Influence of the interviewer on the accuracy of survey results. J. Amer. statist. Ass., 53: $635-59,1958$.

18. KOHN, R. \& WHITE, K.L., ed. Use of health services: an international collaborative study; report of the World Health Organization/International Collaborative Study of Medical Care Utilization. Oxford, Oxford University Press, 1976.

19. KROEGER, A. Errores de respuesta y otros problemas de las encuestas de salud mediante entrevista en los paises en desarrollo. Bol. Ofic. sanit. panamer., 100: 253-82, 1986.

20. LAURELL, A.C.; BLANDO, A.B. \& HEREDIA DUARTE A. El desarrollo urbano y los patrones farmaceuticos. Salud publ. Méx., 19: 397-410, 1977.

21. MANUAL da Classificação Estatística Internacional de Doenças, Lesões e Causas de Óbitos; $9^{2}$ revisão, 1975. São Paulo, Centro da O.M.S. para Classificação de Doenças em Português/Ministério da Saúde/Universidade de São Paulo/Organização Panamericana da Saúde, 1980. 2v.

22. MATTAR, N. Incidência de meningite em diversos grupos da população de Ribeirão Preto, 1978. Ribeirão Preto, 1978. [Dissertação de Mestrado - Faculdade de Medicina de Ribeirão Preto da USP].

23. MEDICI, A.C. Aspectos sócio-econômicos da morbidade no Brasil: uma contribuição aos estudos sobre população e saúde (o caso do Nordeste). Saúde Deb., (30): 40-51, 1990.

24. MORBIDADE hospitalar na rede contratada do INAMPS RADIS-Dados FIOCRUZ, Rio de Janeiro, 6 (12) 1988.

25. RAMOS, M.C. Sintomas respiratórios na população da cidade de Ribeirão Preto, SP (Brasil). Resultados da aplicação de um questionário padronizado. Rev. Saúde públ., S. Paulo, 17: $41-50,1983$.

26. ROSS, D.A. \& VAUGHAN, J.P. Health interview surveys in developing countries: a methodological interview with recommendations for future surveys. London, London School of Hygiene and Tropical Medicine, 1984. (E.P.C. Publication $\mathrm{n}^{2} 4$ ).

27. RUFFINO NETTO, A. \& CARVALHEIRO, J. da R. Doenças do aparelho respiratório e infecciosas e parasitárias na morbidade referida. Rev. Med., Ribeirão Preto, 15 (2): 79 . 83, 1982.

28. WHITE, K.L.; ANDJELKONIC, M.D.; PEARSON, R.J.C.; MARBRY, J.H.; ROSS, A.; SAGEN, O.K. International parisons of medical care utilization. New Engl. J. Med., 277: 516-22, 1967

29. WHITE, K.L.; ANDERSON, D.O.; BICE, T.W.; KALIMO, E.; SCHACH, E. Health care: an international comparison of perceived morbidity, health services resources and use. Int. J, Hlth Serv., 6: 199-218, 1976. 